Copyright by the American Institute of Physics (AIP). Dong, SX; Li, JF; Viehland, D., "Vortex magnetic field sensor based on

ring-type magnetoelectric laminate," Appl. Phys. Lett. 85, 2307 (2004); http://dx.doi.org/10.1063/1.1791732

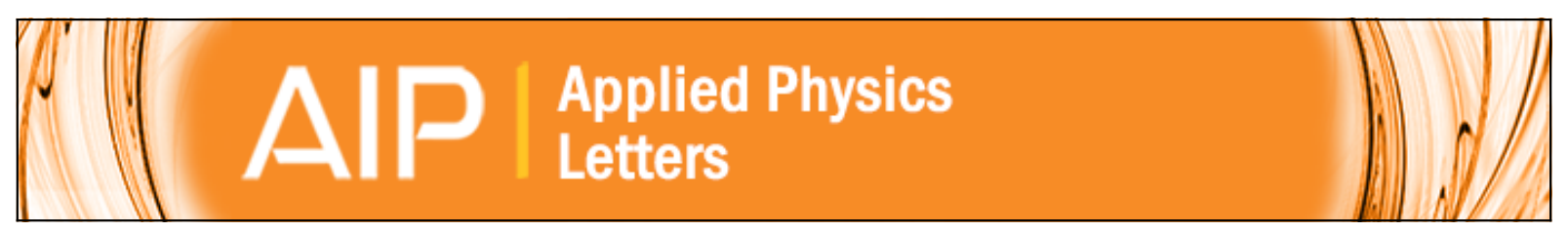

Vortex magnetic field sensor based on ring-type magnetoelectric laminate

Shuxiang Dong, Jie-Fang Li, and D. Viehland

Citation: Applied Physics Letters 85, 2307 (2004); doi: 10.1063/1.1791732

View online: http://dx.doi.org/10.1063/1.1791732

View Table of Contents: http://scitation.aip.org/content/aip/journal/apl/85/12?ver=pdfcov

Published by the AIP Publishing

Over 700 papers \&

presentations on

multiphysics simulation vew now

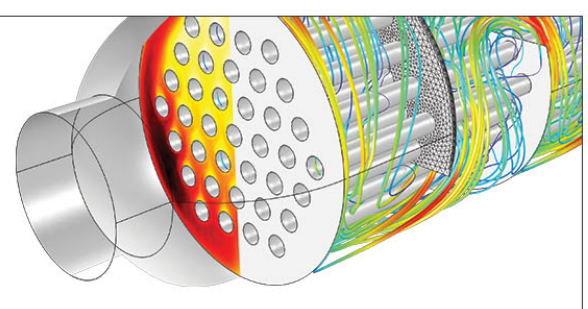

ปே Сロ 


\title{
Vortex magnetic field sensor based on ring-type magnetoelectric laminate
}

\author{
Shuxiang Dong, ${ }^{\text {a) }}$ Jie-Fang Li, and D. Viehland \\ Materials Science and Engineering, Virginia Tech, Blacksburg, Virginia 24061
}

(Received 17 February 2004; accepted 13 July 2004)

\begin{abstract}
It has been found that ring-type magnetoelectric laminate composites of circumferentially magnetized magnetostrictive TERFENOL-D and a circumferentially poled piezoelectric $\mathrm{Pb}(\mathrm{Zr}, \mathrm{Ti}) \mathrm{O}_{3}$ have high sensitivity to a vortex magnetic field. At room temperature, an induced output voltage from this ring laminate exhibited a near-linear response to an alternating current (ac) vortex magnetic field $H_{\mathrm{ac}}$ over a wide magnetic field range of $10^{-9}<H_{\mathrm{ac}}<10^{-3} \mathrm{~T}$ at frequencies between sub-Hz and kHz. (C) 2004 American Institute of Physics. [DOI: 10.1063/1.1791732]
\end{abstract}

The magnetoelectric (ME) effect ${ }^{1}$ in materials which are simultaneously ferromagnetic and ferroelectric has been a research topic in recent years, due to potential energy transduction between magnetic and electrical forms. ME materials of single phase, multiple phases, and laminate composites have been reported. ${ }^{2-16}$ It is known that piezoelectric/ magnetostrictive composites have better ME properties than single-phase materials. ${ }^{2-16}$ Previous investigations have focused on ME laminates whose piezoelectric/magnetostrictive layers are some simple configurations of disk, square, or rectangular shapes. These geometries are only suitable for detection of magnetic fields of constant direction. However, in many situations, there is a need to detect ac rotating or vortex magnetic fields, excited by wires carrying current $I$, such as in power integrated circuits ${ }^{17,18}$ and superconducting films. ${ }^{19}$

In this investigation, a ring-type $\mathrm{ME}$ composite is proposed for vortex magnetic field detection. A ring laminate was made from a $\mathrm{Pb}(\mathrm{Zr}, \mathrm{Ti}) \mathrm{O}_{3}(\mathrm{PZT})$ piezoelectric ring layer (with $m=4$ segments) laminated between two TERFENOL-D $\left(\mathrm{Tb}_{1-x} \mathrm{Dy}_{x} \mathrm{Fe}_{2-y}\right)$ ring layers, as illustrated in Fig. 1. The outer/inter diameters of the rings can be from $3 \mathrm{~mm} / 1 \mathrm{~mm}$ to $25 \mathrm{~mm} / 12 \mathrm{~mm}$, depending upon the wire diameter carrying the ac current $I$. Our ring configuration consisted of circumferentially magnetized TERFENOL-D ring layers and a circumferentially poled piezoelectric ring layer-a $C-C$ ME laminate composite. This configuration intensifies the principle strain/vibration in the circumferential direction of the ring laminate under a vortex magnetic field drive. Our configuration is significantly different than previous ones. ${ }^{2-16}$

In a $C-C$ laminate, because the piezomagnetic and piezoelectric layers are mutually coupled via strain $S(z)$ and stress $T(z)$, application of an ac vortex magnetic field $H_{\mathrm{ac}}$ along the circumferential direction of the magnetostrictive ring layers puts the piezoelectric one into forced oscillation along the same direction. This excites a radial symmetric vibration mode in the piezoelectric, generating a voltage across each segment of the piezoelectric ring layer, via the longitudinal piezoelectric constant $d_{33, p}$. In analysis, we used an equation of motion to couple the magnetostrictive and piezoelectric constitutive relations. By applying Newton's second law of motion to the ring laminate and finding analogous electrical parameters, ${ }^{20,21}$ a magnetoelastoelectric



equivalent circuit (applicable at low frequencies of $f \ll f_{0}$, where $f_{0}$ is the free angular resonance frequency of the laminate) was found, as shown in Fig. 2. The induced ME voltage as a function of an applied ac vortex magnetic field $H_{\mathrm{ac}}$ for the $C-C$ laminate is

$$
V_{\mathrm{ME}}^{C-C}=\beta \frac{\varphi_{m} \varphi_{p}^{2}}{m C_{0} M f_{0}^{2}+\varphi_{p}^{2}} H_{\mathrm{ac}}
$$

where $\beta(V / N)$ is a ratio constant related to dc magnetic bias applied to the ring; $\varphi_{m}$ and $\varphi_{p}$ are the magnetoelastic and elastoelectric coupling factors, respectively; $C_{0}$ is the clamped capacitance of each segment in the piezoelectric ring; and $M$ is the inertial mass of the ring laminate. Equation (1) predicts that $V_{\mathrm{ME}}^{C-C}$ should be a linear function of $H_{\mathrm{ac}}$ and that it should be proportional to both the magnetoelastic and elastoelectric coupling factors.

The ME voltage induced across the PZT ring layer was measured over a broad magnetic field amplitude of $10^{-9}<H_{\mathrm{ac}}<10^{-3} \mathrm{~T}$ in the frequency range of $0.5 \mathrm{~Hz}$ to $2 \mathrm{kHz}$ using a charge amplifier (Kistler Charge Meter, 5015, for preamplifying) and a lock-in amplifier (model SR850 DSP, for ME signal frequency following). A thin long-

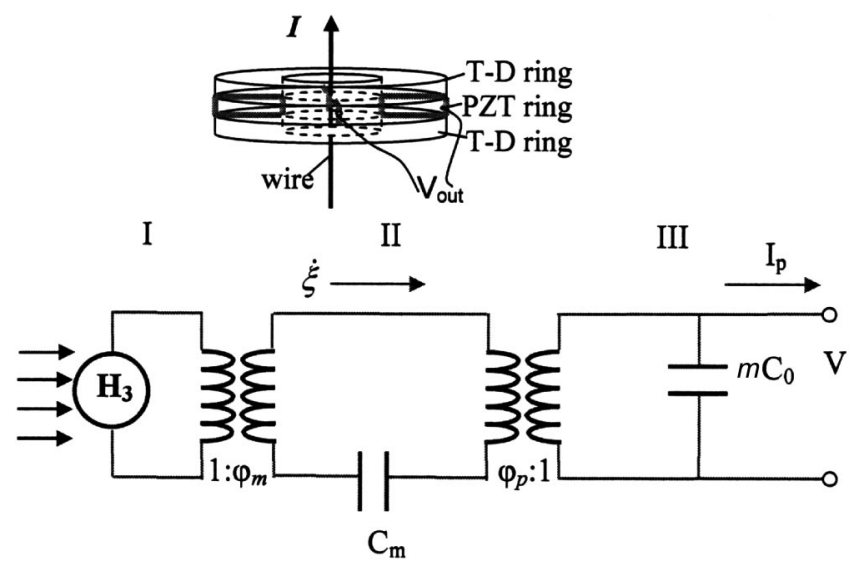

FIG. 1. Vortex magnetic field sensor and its magnetoelastoelectric equivalent circuit at low frequency $\left(f \ll f_{0}\right)$, where I is the magnetic section, II the mechanical section, and III the electric section; $\varphi_{m}=\left(4 \pi A_{m} d_{33, m}\right) / a s_{33}^{H}$ is the magnetoelastic coupling factor; $\varphi_{p}=\left(m A_{p} d_{33, p}\right) / a s_{a s}^{E}$ is the elastoelectric coupling factor; and $C_{0}=\left(1-k_{33 p}^{2}\right)\left(w t_{p} \varepsilon_{33}^{T} / 2 \pi a / m\right)$, is the clamped capacitance of each segment in the piezoelectric ring layer; and $C_{m}=1 / M \omega_{0}^{2}$ is the mechanical compliance of the ring laminate. The vortex magnetic field is excited by a straight wire that is inserted in center of the ring sensor and carrying a current $I$. 


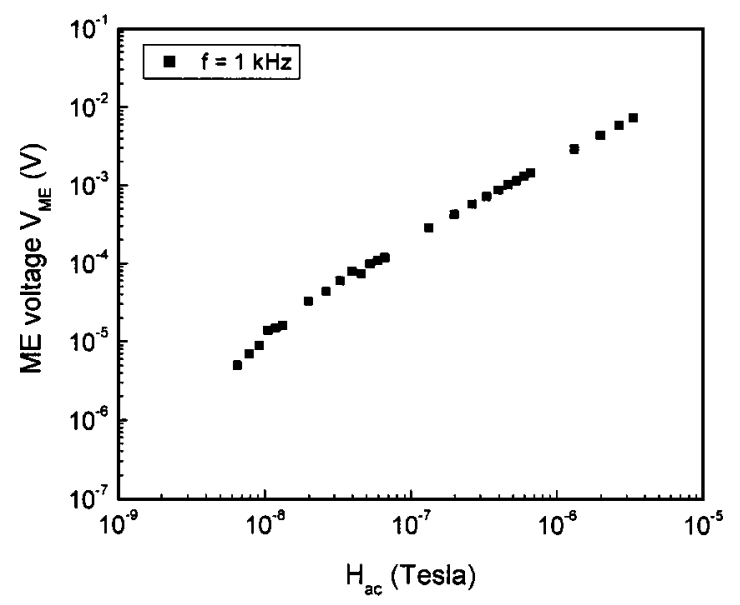

FIG. 2. Induced $\mathrm{ME}$ voltage as a function of magnetic field from $10^{-9}<H_{\mathrm{ac}}<10^{-5} \mathrm{~T}$.

straight wire was inserted at the center of the $C-C$ ring, which generates a small ac vortex magnetic field $H_{\mathrm{ac}}$, via an input ac current $I$

$$
H_{\mathrm{ac}}=\frac{I}{2 \pi a},
$$

where $a$ is mean radial of the $C-C$ ring. Figure 2 shows the measured ME voltage as a function of $H_{\mathrm{ac}}$ in the magnetic field range of $10^{-9}<H_{\mathrm{ac}}<10^{-5} \mathrm{~T}$ at a drive frequency of $1 \mathrm{kHz}$. The induced ME voltage of the $C-C$ ring was found to be a linear function of $H_{\mathrm{ac}}$ over this range, as predicted by Eq. (1). The ME voltage was also found to depend on dc magnetic bias $H_{\mathrm{dc}}$, reflecting the dc-biased piezomagnetic behavior of TERFENOL-D, ${ }^{21}$ which is maximum near the inflection point of the quadratic strain-magnetic field $(\varepsilon$ $-H)$ curve. These measurements were performed at ambient conditions, without magnetic shielding. The results unambiguously demonstrate that the $C-C$ ring laminate has a high sensitivity to small ac vortex magnetic field variations.

The ME voltage was then measured over a magnetic field frequency range of $0.5 \mathrm{~Hz}$ to $2 \mathrm{kHz}$ at a constant magnetic field amplitude of $H_{\mathrm{ac}}=1 \mathrm{Oe}$, as shown in Fig. 3. In Fig. 3, the induced ME voltage can be seen to have an excellent flat response to $H_{\mathrm{ac}}$ in the frequency range of sub-Hz to $\mathrm{kHz}$. The maximum ME voltage using a drive of $H_{\mathrm{ac}}$

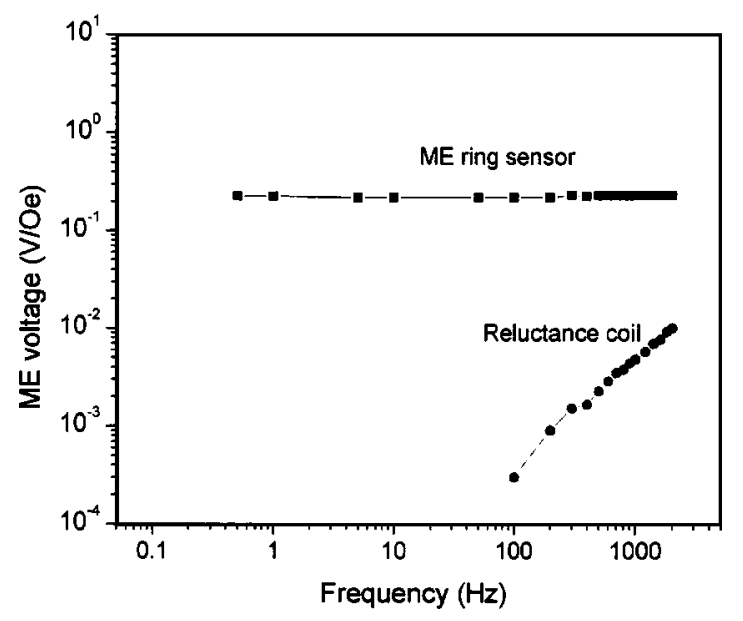

FIG. 3 Induced ME voltage as a function of the ac vortex magnetic field frequency from $0.5 \mathrm{~Hz}$ to $2 \mathrm{kHz}$.
$=1$ Oe amplitude was $\sim 260 \mathrm{mV}$, under a magnetic bias of $H_{\mathrm{dc}}=500$ Oe. This sensitivity is much higher than that of other types of magnetic field sensors. ${ }^{12}$

As a comparison, a toroidal-type variable reluctance coil with $N=100$ turns was also used to detect the ac vortex magnetic field. Figure 3 shows that the induced voltage from a reluctance coil sensor is much smaller (by a factor of 0.1 to 0.01 times) than that of our $C-C$ ME ring sensor. Also, for the reluctance coil, the induced voltage was strongly dependent on the operational frequency. For $f=100 \mathrm{~Hz}$, the flux change rate passing through the coil is so small that the signal detected by the coil disappears into the noise floor. Another alternative vortex sensor is a Hall device; however, its sensitivity is quite low. ${ }^{19}$

Our miniature ring laminate of TERFENOL-D and $\mathrm{Pb}(\mathrm{Zr}, \mathrm{Ti}) \mathrm{O}_{3}$, driven in a $C-C$ mode, has a high sensitivity of $10^{-9} \mathrm{~T}$ to a vortex magnetic field. We believe that this sensitivity limit can be enhanced to $10^{-11} \sim 10^{-12} \mathrm{~T}$ after materials and assembly techniques are improved. This is a significant achievement for a totally passive $(\sim 0 \mu \mathrm{W}$ power consumed) and compact vortex magnetic field sensor that has an output voltage linearly proportional to $H_{\mathrm{ac}}$. It offers opportunities in sensitive vortex magnetic field sensing over wide amplitude and frequency ranges. In particular, our $C-C$ ME sensor has significant potential as an electric current sensing device for power electronic modules. This is because of its advantage of being nonpower consuming (passive) and noncontact; furthermore, by miniaturizing the device to $\leqslant 5 \mathrm{~mm}$ in diameter, the upper limit of the operational frequency range can be increased to hundreds of $\mathrm{kHz}$, suitable for high power conditions.

In summary, a vortex magnetic field sensor based on a miniature ring-type $\mathrm{ME}$ composite of a $\mathrm{Pb}(\mathrm{Zr}, \mathrm{Ti}) \mathrm{O}_{3}$ layer laminated between two TERFENOL-D layers operated in a $C-C$ mode was developed. The results demonstrate: (i) a giant ME voltage of $>260 \mathrm{mV}$ for a $H_{\mathrm{ac}}=1$ Oe; (ii) a high magnetic field sensitivity of $<10^{-9} \mathrm{~T}$, which has potential for $10^{-11} \sim 10^{-12} \mathrm{~T}$ resolution; and (iii) a linear ME voltage response to changes in ac vortex magnetic field over a range of $10^{-9}<H_{\mathrm{ac}}<10^{-3} \mathrm{~T}$ in the frequency range of sub- $\mathrm{Hz}$ to $\mathrm{kHz}$.

This research was supported by the Office of Naval Research under Grant Nos. N000140210340, N000140210126, and MURI N000140110761.

${ }^{1}$ L. D. Landau and E. Lifshitz, Electrodynamics of Continous Media (Pergamon, Oxford, United Kingdom, 1960), p. 119.

${ }^{2}$ M. Avellaneda and G. Harshe, J. Intell. Mater. Syst. Struct. 5, 501 (1994).

${ }^{3}$ T. Wu, J. Huang, Int. J. Solids Struct. 37, 2981 (2000).

${ }^{4}$ K. Mori and M. Wuttig, Appl. Phys. Lett. 81, 100 (2002).

${ }^{5}$ J. Ryu, A. V. Carazo, K. Uchino, and H. Kim, J. Electroceram. 7, 24 (2001).

${ }^{6}$ J. Ryu, A. V. Carazo, K. Uchino, and H. Kim, Jpn. J. Appl. Phys., Part 1 40, 4948 (2001).

${ }^{7}$ J. Ryu, S. Priya, K. Uchino, H. Kim, and D. Viehland, J. Korean Ceram. Soc. 39, 813 (2002)

${ }^{8}$ C.-W. Nan, G. Liu, and Y. Lin, Appl. Phys. Lett. 83, 4366 (2003).

${ }^{9}$ C. W. Nan, L. Liu, N. Cai, J. Zhai, Y. Ye, and Y. H. Lin, Appl. Phys. Lett. 81, 3831 (2002).

${ }^{10}$ M. Avellaneda and G. Harsche, J. Intell. Mater. Syst. Struct. 5, 501 (1994).

${ }^{11}$ G. Srinivasan, E. T. Rasumssen, B. Levin, and R. Hayes, Phys. Rev. B $6 \mathbf{6 5}$, 134402 (2002).

${ }^{12} \mathrm{Y}$. Li and R O Handley (sensors online: http:/sensorsmag.com/articles/ 1000/52/index.htm). 
${ }^{13}$ S. Dong, F. Bai, J. F. Li, and D. Viehland, IEEE Trans. Ultrason. Ferroelectr. Freq. Control 50, 1236 (2003).

${ }^{14}$ S. Dong, J. F. Li, and D. Viehland, Appl. Phys. Lett. 83, 4812 (2003).

${ }^{15}$ S. Dong, J. Cheng, J. F. Li, and D. Viehland, Appl. Phys. Lett. 83, 2265 (2003).

${ }^{16}$ Y. X. Liu, J. G. Wan, J.-M. Liu, and C. W. Nan, J. Appl. Phys. 94, 5111 (2003).

${ }^{17}$ G. Busatto, R. L. Capruccia, F. Iannuzzo, F. Velardi, and R. Roncella,
Microelectron. Reliab. 43, 577 (2003).

${ }^{18}$ J. G. Bai, G.-Q. Lu, and T. Lin, Sens. Actuators, A 109, 9 (2003).

${ }^{19}$ K. Senapati, S. Chakrabaty, L. K. Sahoo, and R. C. Budhani, Rev. Sci. Instrum. 75, 141 (2004).

${ }^{20}$ W. Mason, Physical Acoustics, Principles and Methods, Part A (Academic, New York, 1964), p. 1.

${ }^{21} \mathrm{G}$. Engdahl, Magnetostrictive Materials Handbook (Adcademic, New York, 2000). 